\title{
The Detectors Response of an EDS Spectrometer in Low Energy Spectrum Part
}

Frank Eggert $^{1}$, Ulrich Gernert $^{2}$, Felix Reinauer ${ }^{3}$ and Patrick Camus ${ }^{3}$

${ }^{1}$ AMETEK / EDAX, Mahwah, New Jersey, United States, ${ }^{2}$ Technische Universität Berlin, Berlin, Berlin, Germany, ${ }^{3}$ AMETEK, Mahwah, New Jersey, United States

The response function of Energy Dispersive X-ray Spectrometers (EDS) has been a topic since its inception. New EDS low-energy sensitivity requires the understanding of this spectrum part, especially below $600 \mathrm{eV}$. The known Incomplete Charge Collection (ICC) [1,2] was found to be a phenomenon of lost electrons if photons are absorbed near the detector surface, which causes 'shelf' and 'tail' of the peaks. If the energy of photons is $<600 \mathrm{eV}$, then the charge-loss effect of some detected X-ray morphs into a quantitative problem by causing a peak shift towards lower energies. Recently it was reported that the shift-effect is less strong below $149 \mathrm{eV}$ and even disappears for photons with energies < $99 \mathrm{eV}[3,4]$. Due to the $\mathrm{Si}-\mathrm{L}$ edges (mass absorption coefficient jump) those photons can penetrate deeper into Silicon material with no charge losses and are therefore practically without shift, Figure 1 . But the observed line positions with $\mathrm{Al}-\mathrm{L}$ and $\mathrm{Si}-\mathrm{L}$ are about $5 \mathrm{eV}$ higher than expected. It might be because the pulse processor is able to play an active role on the low-energy side with these very low energy detector signals.

Near-surface detector effects can also affect the energy resolution. It is known that the low-energy peaks are widened in resolution compared to the theoretical calculation based on measured FWHM at Mn-Kalpha. But this effect disappears for very low photon energies also, together with the shift-effect, for similar reasons.

Another interesting topic is the escape peaks at these low energies. This is an incomplete chargegeneration, an X-ray photon escapes from detector with a measurement loss of $1.74 \mathrm{keV}$. It happens with X-rays absorbed comparable deep inside the detector. A usual correction is performed channel by channel [5]. But it is well known that this method reproduces the energy resolution of the parent peaks to the lower energies escape peaks, which is incorrect. The detector signal of an escape-peak is similarly processed as the real X-ray photon signals at same energies. Therefore, the energy resolution of the escape peak(s) is like that for an escape-peak-remaining-energy peak(s) and not that of the parent photon energy peak(s). This deviation is small for higher-energy escape peaks and is normally within the statistics. But this is not true for the very low-energy spectral region. Figure 2a shows a significant deviation between the measured spectrum and calculated escape peaks of Au-M. The escape correction [5] was modified to identify visible escape peaks inside of the calculated escape distribution. These distribution parts will be then extracted and replaced by remodelled escape-lines with same sum counts, but for parents related original peak positions minus $1.74 \mathrm{keV}$, and for the detector resolution at the escape peak energy. But these $\mathrm{x}$-rays are not generated in the top surface layer which require change energy position in the spectrum. Therefore, careful energy accounting must be performed for both the raw energy-depleted x-rays and the full energy measured escape peak x-rays. The energy shift-correction only needs to be applied to the raw $\mathrm{x}$-rays to obtain a proper reconstructed spectrum, Figure $2 \mathrm{~b}$. If the escape-correction is applied directly to the displayed measured spectrum, then it is required to perform escape-correction before the shift-correction will be applied. 

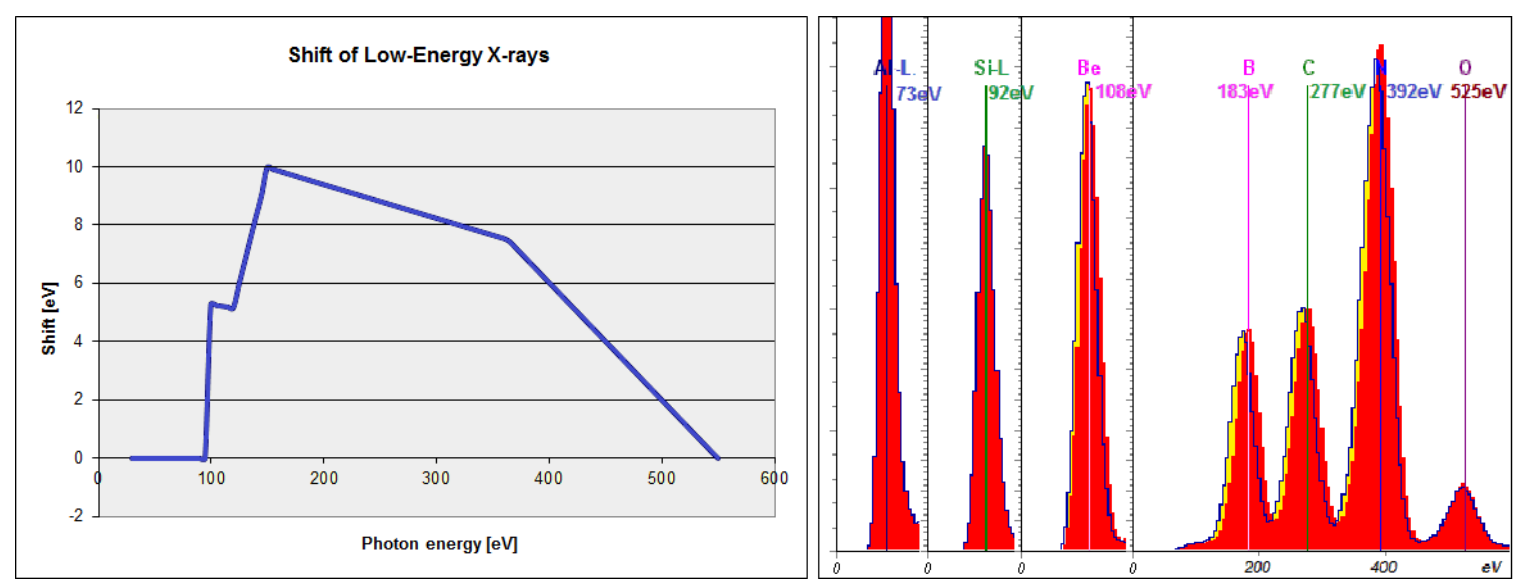

Figure 1. Shift effect of typical SDD detector for measured peaks below $600 \mathrm{eV}$ energy [3].

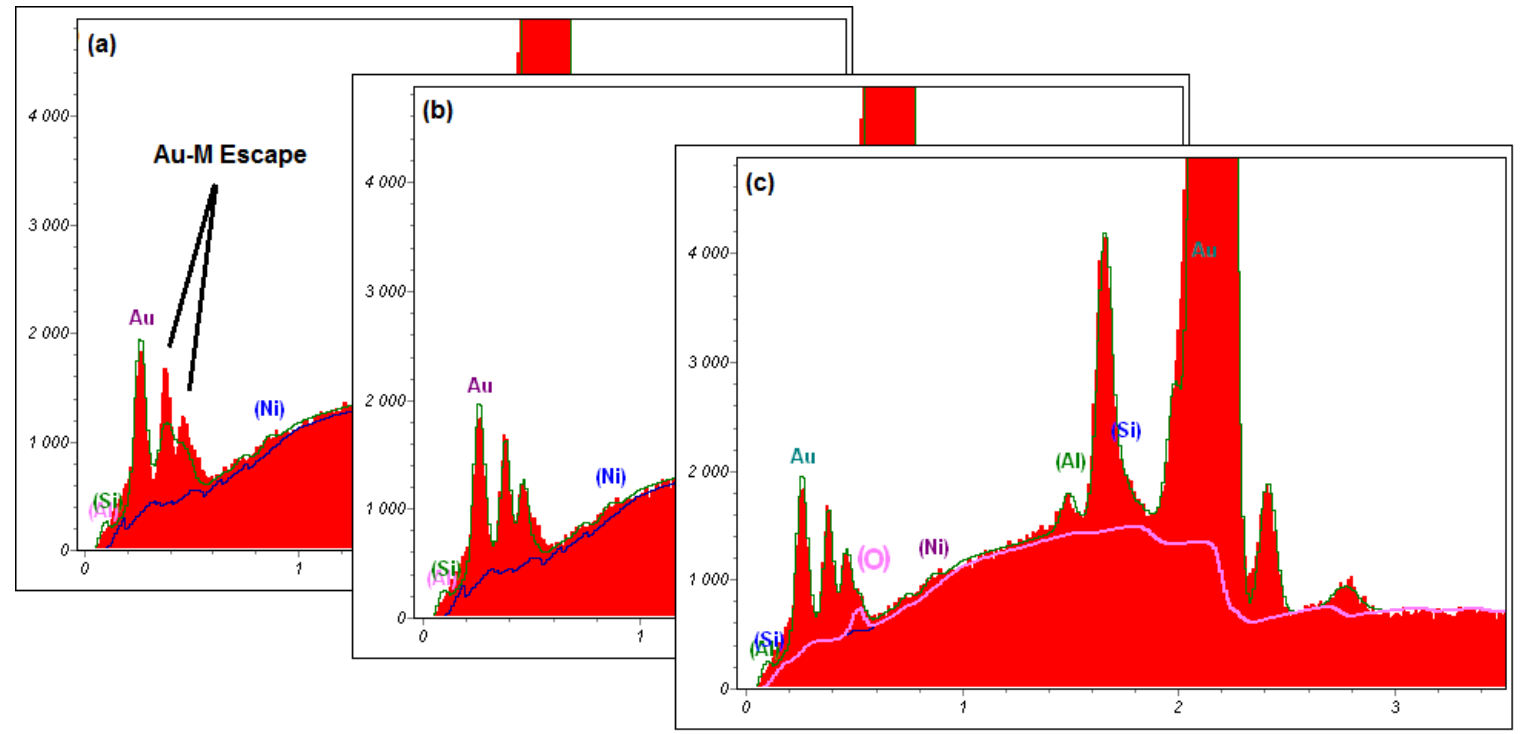

Figure 2. Acquired spectrum of a pure Au standard $(20 \mathrm{kV})$, shift-corrected, in comparison to calculated bremsstrahlung background (blue line) and reconstructed spectrum after complete spectrum deconvolution (green line). The reconstruction contains also all artefacts: pile-up events, shelf, tail and escape. (a) This is with the old escape correction [5]. (b) The reconstruction with modified escape correction for low-energy part fits much better. (c) An Oxygen peak is possible to detect with the modified correction (pink line), which cannot be measured with the old correction.

\section{References}

[1] J Heckel, W Scholz, X-Ray Spectrom. 18 (1987) 181

[2] F Scholze, M Procop, X-Ray Spectrom. 38 (2009) 312

[3] F Eggert, P P Camus, F Reinauer, "Very Low Energy Peak Shifts in EDS Spectra", Proceedings EMAS, Trondheim (2019) to be published

[4] U Gernert, D Berger, "Specifics in EDS spectrum reconstruction at lowest X-ray energies",

Poster Microscopy Conference, Berlin (2019)

[5] F Eggert, X-Ray Spectrom. 19 (1990) 97 